\title{
SÍNDROME DE FOURNIER E SUA POTENCIAL VARIABILIDADE MICROBIOLÓGICA
}

\section{FOURNIER'S SYNDROME AND ITS POTENTIAL MICROBIOLOGICAL VARIABILITY}

\author{
Felicson Leonardo Oliveira Lima1, Fabiana Carneiro de Almeida', Fabiane Silva Reis \\ Góes $^{2}$, Tasciano dos Santos Santa Izabel ${ }^{3}$
}

1 - Graduando de Biomedicina pela Faculdade Nobre de Feira de Santana - FAN - Feira de Santana, Bahia, Brasil

2 - Graduando de Biomedicina pela Unidade de Ensino Superior de Feira de Santana - UNEF - Feira de Santana, Bahia, Brasil

3 - Doutor em Botânica (UEFS), docente da Unidade de Ensino Superior de Feira de Santana - UNEF - Feira de Santana, Bahia, Brasil

Autor para correspondência: E-mail: felicsonleonardo@hotmail.com

\section{RESUMO:}

A Síndrome de Fournier é uma patologia infecciosa que acomete o tecido subcutâneo e a fáscia muscular, sendo considerada como multifatorial. Pode acometer a região perianal e genital, com possibilidade de se alastrar para tecidos adjacentes. Caracteriza-se como uma infecção polimicrobiana, onde as atividades de uma variabilidade de microrganismos podem ocasionar a síndrome. Este estudo objetivou descrever aspectos gerais sobre a doença, os possíveis microrganismos causadores da patologia, bem como a importância da associação entre o diagnóstico clínico, laboratorial e tratamentos adequados. Deste modo, foi realizada uma revisão literária, baseando-se em publicações entre os anos de 2004 a 2019. Conclui-se que a junção entre os fatores de risco como diabetes e etilismo é determinante para o acometimento da doença, sendo o emprego da oxigenioterapia hiperbárica eficaz para o tratamento dessa enfermidade.

PALAVRAS-CHAVE: Grangrena de Fournier; Fascite Necrosante; Endarterite Obliterante.

\section{ABSTRACT:}

Fournier Syndrome is an infectious pathology that affects the subcutaneous tissue and muscle fascia and is considered multifactorial. It can affect the perianal and genital region, possibly spreading to adjacent tissues. It is characterized as a polymicrobial infection, where the activities of a variability of microorganisms can cause the syndrome. This study aimed to describe general aspects about the disease, the possible pathology-causing microorganisms, as well as the importance of the association between clinical, laboratory diagnosis and appropriate treatments. Thus, a literature review was carried out, based on publications from 2004 to 2019. It is concluded that the association between risk factors such as diabetes and alcoholism is determinant for the disease, and the use of hyperbaric oxygen therapy effective for the treatment of this disease.

KEY WORDS: Fournier Grangrena; Necrotizing Fasciitis; Endarterite Obliterating.

\section{INTRODUÇÃO}

A Síndrome de Fournier é uma infecção polimicrobiana a qual foi mencionada pela primeira vez por Baurienne no ano de 1764 (CRUZ, 2016). Em 1883, Alfred Fournier (18321914) nomeou inicialmente a infecção como gangrena idiopática fulminante, a qual possui capacidade de destruição da área infectada (MEHL et al., 2010). Os termos Síndrome de 
Fournier (SF) ou Gangrena de Fournier (GF) foram aplicados para os processos infecciosos característicos que acometiam a região perineal (COSTA et al., 2004; KAUFMANN, 2015). A SF é uma infecção do tecido subcutâneo e da fáscia muscular, a qual é considerada como multifatorial, sendo associada frequentemente a comorbidades, tais como: diabetes mellitus, etilismo, hipertensão, obesidade, tabagismo e o uso de imunossupressores (SILVA et al., 2019). A realização de procedimentos cirúrgicos, utilização de fístulas uretrais e de cateter também podem desencadear a patologia (SANTOS et al., 2015). Essa patologia pode acometer a região perianal, perineal e genital, com possibilidade de se alastrar para regiões proximais. Acomete raramente mulheres e crianças em qualquer faixa etária, com maior frequência em homens acima dos 50 anos de idade (MOREIRA et al., 2017).

As manifestações clínicas mais comuns incluem a apresentação de febre, dor, hiperemia, a qual evolui pra necrose, crepitação e saída de secreção purulenta e fétida, havendo a possibilidade de evoluir para sepse e morte. Quando relacionado ao trato urogenital, variadas são as formas de aquisição desta infecção, podendo ser destacado a sondagem visceral e cateterização uretral traumática (TOLEDO et al., 2012; RUBIO, 2019).

A fisiopatologia pode ser determinada como idiopática (DORNELAS et al., 2012). A endarterite obliterante é caracterizada como fator inicial, a qual evolui para uma isquemia e trombose dos vasos, dificultando a passagem de oxigênio e nutrientes ao tecido resultando em necrose (SANTOS et al., 2018). Depois de acometido por necrose a microbiota normal da pele pode adentrar ao tecido. A ação de bactérias anaeróbias e aeróbias, a isquemia tecidual e a hipóxia, resultam na diminuição do metabolismo no tecido e aumento da disseminação de microrganismos facultativos, os quais se suprem das fontes de energia celular e passam a produzir hidrogênio e nitrogênio, esses gases culminam na crepitação, que pode acontecer entre 48 a 72 horas após início da infecção (CRUZ, 2016).

A Síndrome de Fournier é caracterizada como uma fascite necrosante, onde, quando realizada cultura do exsudato de suas lesões, pode ser observada a presença de bactérias, as quais normalmente não apresentam patogenicidade, sendo patogênicas em condições favoráveis (AZEVEDO et al., 2016). As bactérias que podem estar envolvidos nesse tipo de lesão são as aeróbias gram positivas e gram negativas assim como anaeróbias, e outros microrganismos (MOREIRA et al., 2017).

O presente artigo tem como objetivo descrever aspectos gerais sobre a Síndrome de Fournier, os microrganismos associados, bem como a importância da associação entre o diagnóstico clínico, laboratorial e tratamentos adequados. 


\section{METODOLOGIA}

Foi realizada uma revisão de literatura, nas bases de dados SciELO, Bireme e Lilacs, através de artigos publicados entre os anos de 2004 a 2019, nas línguas inglesa, espanhola e portuguesa. Os descritores utilizados nas buscas foram: Gangrena de Fournier, Fascite Necrosante e Endarterite Obliterante. Foram encontrados 200 artigos vinculados à temática, sendo selecionados 20 artigos. Os critérios de inclusão foram artigos publicados dentro do período estipulado e que demonstravam os microrganismos causadores da patologia, excluindo-se as publicações repetidas que surgiram em mais de uma base de dados.

\section{RESULTADOS E DISCUSSÃO}

\subsection{Microrganismos envolvidos}

Nas culturas de pacientes com SF podem ser encontrados vários microrganismos responsáveis pela patogenia, alguns desses, são bactérias que habitualmente não apresentam potencial de patogenicidade, mas quando em condições favoráveis podem ser indutores da enfermidade (MOREIRA et al., 2017).

Assim, Rampelotto et al., (2014) demonstram que as lesões necrotizantes podem ser subdivididas em duas classes, a primeira ocasionada principalmente pelo Streptococcus agalactiae e a segunda, onde se destaca o Streptococcus pyogenes, no entanto, ainda assim pode acontecer uma associação entre Streptococcus pyogenes e Staphylococcus aureus e/ou outras bactérias oportunistas.

As Bactérias Gram negativas aeróbicas também são encontradas na SF, tais como: Pseudomonas aeruginosa, Proteus mirabilis, Escherichia coli, Klebsiella pneumoniae (MAURO, 2011; AZEVEDO et al., 2016). Outras espécies presentes podem ser representadas pelos: Bacteroides fragilis, Bacteroides melaninogenicus, cocos Gram positivos e Clostridium (não perfringens) (CRUZ, 2016).

Pacios et al., (2004) descreve um caso raro de Gangrena acometida por Streptocccus agalactiae de forma secundária, em um paciente alcoólatra com diabetes, sendo o procedimento de cateterismo uretral desencadeador da doença. Dornelas et al., (2012) sugerem os fungos como possíveis promotores da Síndrome de Fournier. Louro et al., (2019) apontam a infecção com possibilidade de causa por Enterocccus faecalis, 
Acinetobacter baumannii, Streptococcus pyogenes, Enterococcus faecium, Enterococcus cloacae, Bacteroides fragilis, Corynebacterium, Candida albicans e Aspergillus fumigatus.

Outros microrganismos que podem estar envolvidos nesse tipo de lesão são as bactérias aeróbias gram positivas e gram negativas assim como anaeróbias, vibriões e fungos (AZEVEDO et al., 2016; MOREIRA et al., 2017). Os microrganismos mais comuns associados à Síndrome de Fournier são listados na tabela 1.

TABELA 1: MICRORGANISMOS ENVOLVIDOS NA SÍNDROME DE FOURNIER

\section{Microrganismos Envolvidos}

Streptococcus agalactiae,

Streptococcus pyogenes,

Staphylococcus aureus, Streptococcus

viridans, Streptococcus fecalis;

Staphylococcus epidermidis,

Bacteroides fragilis,

Bacteróides melaninogenicus;

Escherichia coli,

Pseudomonas pyocyaneus, Klebsiella

pneumoniae;

Pseudomonas aeruginosa,

Proteus mirabilis;

Clostridium spp e

Fungos filamentosos;

Acinetobacter baumannii,

Enterococcus cloacae,

Corynebacterium,

Candida albicans,

Aspergillus fumigatus,

Corynebacterium,

Enterobacter cloacae,

Klebsiela cloacae;

Clostridium septicum;

Bacteroides fragilis,

Bacteroides melaninogenicus,

Clostridium (não perfringens);
Referências

(RAMPELOTTO et al., 2014)

(MOREIRA et al., 2017)

(MAURO, 2011)

(AZEVEDO et al., 2016)

(DORNELAS et al., 2012)

(LOURO et al., 2019)

Fonte: Elaborada pelos autores

\subsection{Diagnóstico}

O diagnóstico é na grande maioria das vezes clínico, confirmado com os achados cirúrgicos (liquefação da gordura subcutânea, falta de sangramento e mínima aderência do 
tecido subcutâneo) e requer caráter de precocidade (SOARES et al., 2008).

O diagnóstico microbiológico está relacionado à identificação do agente causal, sendo realizada uma bacterioscopia, com objetivo de caracterizar a espécie (TOLEDO et al., 2012). Em conjunto, é realizado o antibiograma, na tentativa de avaliar a sensibilidade ou resistência do microrganismo encontrado (SOARES et al, 2008).

Dentre as alterações laboratoriais podem ser notados quadros anêmicos, leucocitose, aumento da Proteína C Reativa (PCR), hipocalcemia, hiperglicemia, além de elevação da creatinofosfoquinase (CPK) e da velocidade de hemossedimentação (VHS) (SOARES et al., 2008).

Exames de imagem como a radiografia convencional podem detectar a presença de ar nas partes moles (PORTILLO et al., 2019). O ultrassom, bem como a tomografia computadorizada e a ressonância magnética também são úteis e auxiliam determinando a extensão da gangrena (TOLEDO et al., 2012).

A Biópsia da fáscia é de grande relevância diagnóstica, sendo considerada padrãoouro, podendo ser realizada durante o desbridamento (SOARES et al., 2008). Nos exames histopatológicos, alterações correlacionadas com a necrose podem ser notadas, como a infiltração de polimorfonucleares e edema da derme reticular (SOARES et al., 2008).

\subsection{Tratamento}

Diversas metodologias para a antibioticoterapia podem ser utilizadas devido ao amplo espectro bacteriológico associado à doença (MOREIRA et al., 2017). Na grande maioria das vezes o diagnóstico é clínico com administração medicamentosa logo após a conclusão do laudo sem aguardar o resultado da cultura e testes de sensibilidade, podendo ser alterados posteriormente, conforme evolução e resultados da microbiologia (SOARES et al., 2008; REIS, 2012).

O tratamento preferencial deve cobrir atuação de microrganismos Gram positivos da pele, Gram negativos do trato genito-urinário e entérico, bem como anaeróbios (MEHL et al., 2010). Recomenda-se que haja uma associação do desbridamento cirúrgico como linha inicial e tratamento clínico com antibioticoterapia (DORNELAS et al., 2012).

O desbridamento cirúrgico é um método efetivo para remoção do tecido necrótico, sendo possível remover todo o tecido necrosado nesse procedimento, evitando assim que a infecção se espalhe (MEHL et al., 2010). A drenagem de secreção purulenta possibilita redução do edema e promove remoção mecânica dos microrganismos presentes no tecido 
infectado (SANTOS, 2013; SANTOS et al., 2018).

A oxigenoterapia hiperbárica possui grande eficiência, uma vez que auxilia na redução da atividade de endotoxinas e redução da extensão da necrose (MEHL et al., 2010; AZEVEDO et al., 2016). Os seus benefícios estão destinados à remoção do exsudato, estímulo a angiogênese e redução da contaminação bacteriana (DORNELAS et al., 2012).

\section{CONCLUSÂO}

A Síndrome de Fournier é uma patologia de causa idiopática na maioria dos casos, sendo a associação entre os fatores de risco determinante para o acometimento. $O$ diagnóstico com intervenção rápida influencia na melhoria dos doentes, as taxas de mortalidade mostram-se elevadas em casos de diagnóstico retardados, uma vez que a SF pode gerar sepse logo no início do quadro infeccioso. A desnutrição e condições imunossupressoras, como o HIV e leucemias, são influxos para a piora do prognóstico.

Os microrganismos que ocasionam essa patologia podem atuar como causa principal ou como oportunistas. Produzem toxinas e enzimas capazes de promover necrose ao tecido. No processo de cateterismo (cateterização uretral traumática), microrganismos podem contaminar o material, ou ainda, bactérias da microbiota podem ser arrastadas durante o procedimento. Além disso, à técnica pode promover lesão tecidual, possibilitando a penetrabilidade de microrganismos, os quais, em condições favoráveis, promoverão a infecção.

Na grande maioria das vezes o diagnóstico se dá na forma clínica, com confirmação e terapia dependente da análise laboratorial, sendo o diagnóstico precoce importante para o tratamento da doença. O uso da antibioticoterapia de largo espectro é geralmente a metodologia aderida. Atualmente, o emprego da oxigenioterapia hiperbárica tem sido utilizada, ajudando na manutenção das taxas de oxigênio para o metabolismo celular, melhorando cicatrização e defesa.

\section{REFERÊNCIAS}

AZEVEDO, Cassius Clay SF et al. Síndrome de Fournier: Um artigo de revisão. Revista Eletrônica do UNIVAG, n. 15, p. 70-78 2016.

COSTA, Izelda Maria Carvalho et al. Fasciíte necrosante: revisão com enfoque nos aspectos 
dermatológicos / Necrotizing Fasciitis: new insights with a focus on dermatological aspects. Anais Brasileiros de Dermatologia, Rio de Janeiro, v. 79, n. 2, p. 211-224, 2004.

CRUZ, Ronny Anderson de Oliveira; ANDRADE, Lidiane Lima; ARRUDA, Aurilene Josefa Cartaxo Gomes. Produção Científica Sobre Gangrena de Fournier e os Cuidados de Enfermagem: Revisão Integrativa. Revista de Enfermagem UFPE on line, Recife, v. 10, Supl. 5, p. 4329-4335, 2016.

DORNELAS, Marilho Tadeu et al. Síndrome de Fournier: 10 anos de avaliação. Revista Brasileira de Cirurgia Plástica, v. 27, n. 4, p. 600-604, 2012.

KAUFMANN, Judith A; RAMPONI, Denise. Recognition of Risk Factors and Prognostic Indicators in Fournier's Gangrene. Critical Care Nursing Quarterly, v. 38, n. 2, p. 143-153, 2015.

LOURO, João Mendes et al. Fournier Gangrene: experience of a plastic surgery department, Acta Médica Portuguesa, v. 32, n. 5, p. 368-374, 2019.

MOURO, Victor. Retalho fasciocutâneo de região interna de coxa para reconstrução escrotal na síndrome de Fournier. Revista Brasileira de Cirurgia Plástica, v. 26, n. 4, p. 707709, 2011.

MOREIRA, Daniel Rosa et al. Terapêutica cirúrgica na síndrome de Fournier: relato de caso / Surgical therapy in Fournier syndrome: case report. Revista de Medicina. São Paulo, v. 96, n. 2, p. 116-120, 2017.

MEHL, Adriano Antônio et al. Manejo da gangrena de Fournier: experiência de um hospital universitário de Curitiba. Revista do Colégio Brasileiro de Cirurgiões, v. 37, n. 6, p. 435-441, 2010. PACIOS, José Carlos López et al. Gangrena de Fournier Secundaria a cateterismo uretral. Casos Clínicos. Archivos Españoles de Urología, v. 58, n. 2, p. 167-170, 2005.

PORTILLO, Moyano A et al. Fascitis necrotizante secundaria a úlcera por presión. Revista Clínica Médica Familiar, v. 12, n. 1, p. 24-27, 2019. 
RAMPELOTTO, Roberta Filipini et al. Fasciite necrosante por Streptococcus agalactiae em paciente diabética - relato de caso. Scientia Medica, v. 24, n. 2, v. 182-186, 2014.

REIS, Rodolfo Borges; FILHO, Jose Carlos Souza Trindade; SIMÕES, Fabiano André. Guia Rápido de Urologia - GRU: Sessão São Paulo. Sociedade Brasileira de Urologia. Lemar Livraria e Editora Marina, São Paulo, 1ํㅡㄹ ed, 2012.

RUBIO, Tatiana Vargas; AGUERO, Sofía de Los Ángeles Mora; AGUILERA, Ana Sofía Zeledón. Gangrena de Fournier: generalidades. Revista Médica Sinergia, v. 4, n. 6, p. 100$107,2019$.

SANTOS, Isabel Cristina Ramos Vieira; OLIVEIRA, Regina Célia; Mailton Alves da Silva. Desbridamento cirúrgico e a competência do enfermeiro. Texto \& Contexto Enfermagem, v. 22, n. 1, p. 184-192, 2013.

SANTOS, Joyce Silva et al. Avaliação do Uso de Cateter Urinário e Fatores Relacionados em Unidade de Terapia Intensiva Neonatal. Revista ESTIMA, v.13 n.1, p. 17-26, 2015.

SANTOS, Djoney Rafael et al. Perfil dos pacientes com gangrena de Fournier e sua evolução clínica. Revista do Colégio Brasileiro de Cirurgiões, v. 45, n. 1, p. 14-30, 2018.

SILVA, Viviane de Deus et al. Fournier's Syndrome recurrence: a case report. Revista de Patologia do Tocantins, v. 6, n. 2, p. 35-37, 2019.

SOARES, Thiago Horta et al. Diagnóstico e tratamento da Fasciíte Necrosante (FN): relato de dois casos / Necrotizing Fasciitis (NF) diagnosis and treatment: two cases report. Revista Médica de Minas Gerais. Minas Gerais, v. 18, n. 2, p. 136-140, 2008.

TOLEDO, Alexandre Sousa et al. A atuação do biomédico no diagnóstico da síndrome de fournier. Ensaios e Ciência: Ciências Biológicas, Agrárias e da Saúde, v. 16, n. 4, p. 171182, 2012. 